\title{
Oral azithromycin in extended dosage schedule for chronic, subclinical Chlamydia pneumoniae infection causing coronary artery disease: a probable cure in sight? Results of a controlled preliminary trial
}

\section{Jaideep Dogra}

Poly Clinic, Central Government Health Scheme, Jaipur, Rajasthan, India

Video abstract

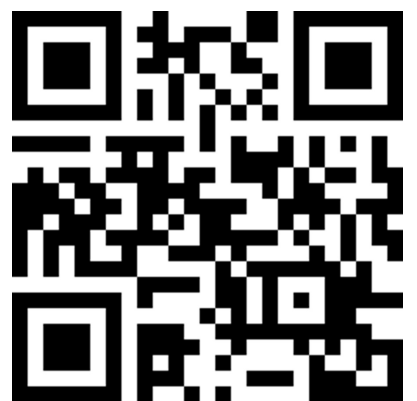

Point your SmartPhone at the code above. If you have a QR code reader the video abstract will appear. Or use: http://dvpr.es//CCBTo

Correspondence: Jaideep Dogra Shivashakti, C-I I-JDA, Malviya Nagar, Jaipur 302017, India

Tel +9l 9829052013

Fax +9। 14। 2523723

Email jd_4ul958@yahoo.com
This article was published in the following Dove Press journal:

International Journal of General Medicine

7 June 2012

Number of times this article has been viewed

Purpose: Two mega trials have raised the question as to whether the hypothesis that infection plays a role in atherosclerosis is still relevant. This controlled preliminary trial investigated an extended dose of azithromycin in the treatment of Chlamydia pneumoniae infection causing coronary artery disease (CAD).

Patients and methods: Forty patients with documentary evidence of CAD were screened for immunoglobulin $\mathrm{G}$ titers against $C$. pneumoniae and grouped into either the study group (patients with positive titer, $n=32$ ) or control group (patients with negative titer, $n=8$ ). Cases who met inclusion criteria could not have had coronary artery bypass graft surgery or percutaneous coronary intervention in the preceding 6 months. Informed consent was obtained from every patient. Baseline blood samples were analyzed for red blood cell indices, serum creatinine, and liver function tests, and repeated every 2 months. A primary event was defined as the first occurrence of death by any cause, recurrent myocardial infarction, coronary revascularization procedure, or hospitalization for angina. Patients in the study group received $500 \mathrm{mg}$ of oral azithromycin once daily for 5 days, which was then repeated after a gap of 10 days (total of 24 courses in the 1-year trial period). The control group did not have azithromycin added to their standard CAD treatment.

Results: In the study group, 30 patients completed the trial. Two patients had to undergo percutaneous coronary intervention in the initial first quarter of the 1-year trial period. In the control group, one patient died during the trial, one had to undergo coronary artery bypass graft surgery, and one had percutaneous coronary intervention.

Conclusion: The patients tolerated the therapy well and there was a positive correlation between azithromycin and secondary prevention of CAD.

Keywords: azithromycin, Chlamydia pneumoniae, coronary artery disease

\section{Introduction}

Of the approximately 600 million bacteria inside and outside of the human body, the majority are commensal. Despite the widespread use of potent antiplatelet agents and statins, the rate of recurrent ischemic events within 2-6 months after an initial episode of acute myocardial infarction and unstable angina remains high $(12 \%-20 \%) \cdot{ }^{1-3}$ The first association between microorganisms and coronary artery disease (CAD) came from a study in Finland, which showed that high titers of immunoglobulin A and G (IgG) 
antibodies to Chlamydia pneumoniae occurred significantly more often in men with myocardial infarction and chronic coronary artery disease (CAD) than in age-matched randomly selected controls. ${ }^{4}$ While the serological data elicited some general interest in the topic, it was the unexpected finding by Shor et al that created much interest. ${ }^{5}$ Using electron microscopy, they noticed chlamydia-like structures in the walls of coronary arteries obtained at necropsy. These findings were confirmed by polymerase chain reaction in an international collaborative study, ${ }^{5}$ and this was followed by further confirmation. ${ }^{6}$

The possibility of the role of $C$. pneumoniae in the pathogenesis of CAD was further strengthened by an animal study in which intranasal infection of rabbits with C. pneumoniae was shown to induce or accelerate atherosclerosis, ${ }^{7}$ and then further still by a study in which azithromycin prevented the acceleration of atherosclerosis induced by $C$. pneumoniae in rabbits. ${ }^{8}$

Since then, evidence has been accumulating on the role of C. pneumoniae - a species of Gram-negative, obligate, intracellular pathogen which commonly causes a spectrum of lower and upper respiratory tract disease in humans and has been implicated in the etiology/pathogenesis of atherosclerosis. Although there is evidence that C. pneumoniae is associated with atheromatous lesions, it is difficult to resolve whether it is a primary cause of disease or a secondary invader. Is this the germ theory of disease revisited? Is $C$. pneumoniae an innocent bystander in CAD? The possibility that chronic inflammatory/degenerative diseases previously thought to be noninfectious may indeed be infectious and thus treatable with antibiotics is dramatically exemplified by the response of peptic ulcer disease to antibiotic therapy directed against Helicobacter pylori $i^{9}$ and chronic plaque psoriasis to antibiotic therapy directed against Streptococcus pyogenes. ${ }^{10}$ The detection of $C$. pneumoniae in young subjects suggests the possibility that it has a primary role in the etiology of atherogenesis, but does not prove it. ${ }^{11}$ Still, the presence of C. pneumoniae in atherosclerotic lesions raises the strong possibility that antichlamydial antibiotic treatment might have a favorable effect on the course of CAD.

The WIZARD (Weekly Intervention with Zithromax for Atherosclerosis and its Related Disorders) study failed to find a long-term benefit, even though a favorable trend was noted during and shortly after 3 months of azithromycin treatment. ${ }^{12}$ More recent trials, ACES (Azithromycin and Coronary Events Study) ${ }^{13}$ and PROVE IT-TIMI 22 (Pravastatin Or Atorvastatin Evaluation and Infection Therapy), ${ }^{14}$ also reported a negative outcome. However, these studies do not disprove the hypothesis that chronic infection with C. pneumoniae is atherogenic, and their negative outcome may be either due to inadequate dosage of azithromycin or an advanced stage of CAD. ${ }^{15}$ Had the negative outcome been due to an advanced stage of the disease, no significant antibiotic protection - even during the initial 6 months of the trial - would have been observed, so what probably went "off target" in the WIZARD trial was the inadequate regimen of azithromycin.

As chronicity is a hallmark of $C$. pneumoniae infection which is susceptible to macrolides, its trial with an optimum dose is still justified, especially when taking the public health importance of CAD into consideration. ${ }^{13}$ Thus, oral azithromycin was chosen, due to its antichlamydial efficacy, in an extended dosage schedule. ${ }^{16-18}$

\section{Patients and methods}

Forty patients with documentary evidence of CAD (including stable angina, unstable angina, and $>3$ months postmyocardial infarction) were enrolled in the present trial (Table 1). IgG titers of C. pneumoniae were measured in all patients by enzyme Immuno-Assay. Those who met the inclusion criteria could not have had a coronary artery bypass graft or percutaneous coronary intervention in the preceding 6 months nor received antibiotics in the preceding 3 months.

After a screening visit at which C. pneumoniae antibody titers were measured, patients with an IgG titer of

Table I Baseline characteristics of patients

\begin{tabular}{lll}
\hline Characteristics & $\begin{array}{l}\text { On azithromycin } \\
(\mathbf{n}=\mathbf{3 2})\end{array}$ & $\begin{array}{l}\text { Control } \\
(\mathbf{n}=\mathbf{8})\end{array}$ \\
\hline Mean age & 56 years & 60 years \\
Sex (male:female) & $26: 6$ & $6: 2$ \\
Cardiac risk factors & & \\
$\quad$ Current smokers & 4 & - \\
$\quad$ Dyslipidemia & 32 & 6 \\
Family history & 7 & 2 \\
$\quad$ Diabetes mellitus & 6 & 1 \\
Hypertension & 20 & 5 \\
Medical history & & \\
$\quad$ Angina & 16 & 3 \\
Congenital heart failure & 3 & 1 \\
Ejection fraction $<35 \%$ & 8 & 3 \\
Last myocardial infarction & 14 months & 12 months \\
Concomitant medicine & & \\
$\quad$ Aspirin/clopidogrel & 27 & 6 \\
ACE inhibitor & 20 & 7 \\
Statin & 22 & 7 \\
B-blocker & 20 & 3 \\
Calcium channel block & 10 &
\end{tabular}

Abbreviation: ACE, angiotensin-converting enzyme. 
C. pneumoniae of 1:16 or greater were assigned to receive oral azithromycin (ie, the study group). Those subjects with a negative titer (or titer less than 1:16) were assigned to the control group and did not have azithromycin added to the already prescribed standard CAD treatment.

Baseline blood samples were analyzed for red blood cell indices, serum creatinine, and liver function tests, and repeated every 2 months. C-reactive protein estimation was done initially and at the end of the 1-year trial period. Informed consent was obtained from all patients.

A primary event was defined as the first occurrence of death by any cause, recurrent myocardial infarction, coronary revascularization procedure (coronary artery bypass graft surgery or percutaneous coronary intervention), or hospitalization for angina.

Patients in the study group received $500 \mathrm{mg}$ of oral azithromycin once daily for 5 days, which was repeated after a gap of 10 days (total of 24 courses in the 1-year trial period). This is a comparable dose to one that was recently used to effectively treat chronic plaque psoriasis. ${ }^{19}$ Long-term azithromycin therapy has been well tolerated in previous trials with minor gastrointestinal side effects (eg, nausea and diarrhea). ${ }^{19,20}$ A statistical $\chi^{2}$ test was applied.

\section{Results}

Of the 32 patients in the study group receiving azithromycin in addition to their normal CAD treatment, 30 patients completed the 1-year trial period without any untoward event. The remaining two patients had to undergo percutaneous coronary intervention in the initial first quarter of the 1 -year trial period.

In the control group, one died during the trial, one had to undergo coronary artery bypass graft surgery, and one had a percutaneous coronary intervention.

No significant association between $C$. pneumoniae titers and outcome of treatment results was observed. In addition, no association between $C$. pneumoniae titers and the likelihood of developing a primary event was seen within the group of patients in the study and control groups.

C-reactive protein was positive in 15 patients in the study group, which became negative in twelve patients after the 1 -year trial period. No difference was noted in the three C-reactive protein-positive cases in the control group.

Mild gastric intolerance and mild diarrhea were reported in six patients who did not require discontinuation of therapy. Remaining patients tolerated the therapy well.

The data were analyzed using a $\chi^{2}$ test. There was a significant positive correlation between the administration of azithromycin and secondary prevention of CAD $\left(\chi^{2}=7.006\right.$; degrees of freedom $=1 ; P<0.01)$.

\section{Discussion}

Two large, high-quality, and convincingly negative clinical trials for the secondary prevention of CAD, WIZARD ${ }^{12}$ and $\mathrm{ACES},{ }^{13}$ raised the question as to whether the hypothesis that infection plays a role in atherosclerosis is still relevant. ${ }^{15}$

Evidence that vascular inflammation is an important mechanism involved in all stages of atherogenesis continues to accumulate. Such evidence has legitimately raised the question of whether infection is one of the inflammatory stimuli that operate in the pathophysiology of atherothrombosis - either locally, within vascular tissue, or systemically - through inflammatory mediators. The web of causation in a multifactorial disease does not imply that CAD cannot be controlled unless the entirety of multiple causes or chains of causation, or at least a number of them, are appropriately controlled or removed. This is not the case. Rather, sometimes removal or elimination of only one link or chain may be sufficient to control a disease provided that the link is sufficiently important in the pathogenesis. Thus, in a multifactorial event like $\mathrm{CAD}$, individual factors are by no means of equal priority. The relative importance of these factors may be expressed in terms of relative risk. A metaanalysis of serological studies has upheld the association between $C$. pneumoniae antibody (IgG) and $\mathrm{CAD},{ }^{21}$ and overall the relative risk of disease occurring in patients with C. pneumoniae antibody is $2-2.6$ times greater than in those without antibody. ${ }^{11,22}$

Ten clinical trials testing antibiotics for the secondary prevention of CAD have been published, ${ }^{13}$ as well as a few others on its role in carotid, ${ }^{23}$ aorta, ${ }^{24}$ and peripheral artery ${ }^{25}$ occlusions. Of these, only the WIZARD trial was adequately powered to give definitive results. ${ }^{12}$ In the WIZARD trial, azithromycin treatment was given as $600 \mathrm{mg}$ once weekly for 2-12 weeks after an initial dose of $600 \mathrm{mg} /$ day for 3 days in the first week. ${ }^{12}$ Its results were similar to the findings of ACES (in which participants were randomly assigned to receive either a $600 \mathrm{mg}$ azithromycin tablet or a matching placebo tablet once weekly for 1 year $)^{13}$ in that they also failed to support the use of antibiotic therapy for CAD. The WIZARD trial, however, did report some antibiotic protection from events during the first 6 months of the trial - a result not repeated by ACES. ${ }^{13}$

In regards to the clinical trials of antibiotic therapy directed at atherosclerotic changes in the carotid arteries, ${ }^{23}$ peripheral arteries, ${ }^{25}$ and the aorta, ${ }^{24}$ each trial reported that 
antibiotic therapy had a significant favorable effect on the progression of disease. These positive results may be considered preliminary; however, if repeated in larger mega trials, they will offer convincing evidence suggesting $C$. pneumoniae is involved in the pathogenesis of atherosclerotic occlusive disease. $^{13}$

The relationship between psoriasis and myocardial infarction has appeared in a recent publication where it was concluded that psoriasis may confer an independent risk of myocardial infarction, and the relative risk was greatest in young patients with severe psoriasis $(3.1 \%) .{ }^{26}$ Recently, it was hypothesized that chronic, subclinical infection of the throat with group A streptococci is the sole etiology involved in the development of psoriatic lesions. ${ }^{10}$

Several mechanisms have been suggested by which infection could contribute to atherogenesis. These include cytopathologic effects of infection on endothelial and smooth muscle cells causing endothelial dysfunction, ${ }^{27,28}$ formation of circulatory toxins or immune complexes that could deposit on vessel walls and elicit an inflammatory response, ${ }^{29}$ and induction or alterations in serum lipid metabolism. ${ }^{30}$ Results of laboratory studies have been cited to support all three mechanisms. ${ }^{22}$

As the rate of a recurrent ischemic event is as high as $12 \%-20 \%$ after an initial episode of acute myocardial infarction/unstable angina, only two patients in the study group $(6.3 \%)$ needing to undergo percutaneous coronary intervention in the initial 3 months of the 1-year study period certainly suggests a significant beneficial role of azithromycin in extended dosage in the treatment of CAD. These two patients who developed a primary event probably had an advanced CAD or the serum levels of the antibiotic were not sufficient to control their disease progression. The length of treatment to choose for this study was difficult to determine. Knowledge of the biology of chronic $C$. pneumoniae infection suggests that short-term treatment is inadequate for a lasting benefit. The elementary body form of the organism is the infectious nonreplicating form, and it is not susceptible to the action of antibiotics. Elementary bodies may exist in the body for weeks, or longer, and cause new cellular infection. Although shortterm treatment might kill replicating organisms in atheroma and temporarily reduce inflammation, it would be unlikely to eliminate the organism from the lesion. Thus, in planning the appropriate length of treatment for a trial, safety both for subjects and the community should be considered. In 1998, Grayston recommended 1 year as the treatment period for C. pneumoniae trials. ${ }^{31}$
Azithromycin and $C$. pneumoniae may be the bridge in the therapeutic and etiological lacuna of CAD if results of this preliminary controlled trial are replicated in future mega trials.

\section{Acknowledgments}

The author would like to thank MC Vyas (Statistical Assistant, Field Survey Unit, Central Bureau of Health Intelligence, Jaipur) for his statistical analysis of the data.

\section{Disclosure}

The author reports no conflicts of interest in this work. This research was presented as an oral presentation and published in Proceedings of the 5th International Congress of Cardiovascular Disease; 2009 June 5-7; Kosice, Slovakia.

\section{References}

1. The RISC Group. Risk of myocardial infarction and death during treatment with low dose aspirin and intravenous heparin in men with unstable coronary artery disease. Lancet. 1990;336(8719):827-830.

2. Cannon CP, Weintraub WS, Demopoulos LA, et al. Comparison of early invasive and conservative strategies in patients with unstable coronary syndromes treated with glycoprotein IIb/IIIa inhibitor tirofiban. $N$ Engl J Med. 2001;344(25):1879-1887.

3. Schwartz GG, Olsson AG, Ezckowitz MD, et al. Effects of atorvastatin on early recurrent ischemic events in acute coronary syndromes: the MIRACL study: a randomized controlled trial. JAMA. 2001;285(13):1711-1718.

4. Mattila K. Viral and bacterial infections in patients with acute myocardial infarction. J Intern Med. 1989;225(5):293-296.

5. Shor A, Kuo CC, Patton DL. Detection of Chlamydia pneumoniae in coronary arterial fatty streaks and atheromatous plaques. S Afr Med J. 1992;82(3):158-161.

6. Kuo CC, Shor A, Campbell LA, Fukushi H, Patton DL, Grayston JT. Demonstration of Chlamydia pneumoniae in atherosclerotic lesions of coronary arteries. J Infect Dis. 1993;167(4):841-849.

7. Fong IW, Chiu B, Viira E, Fong MW, Jang D, Mahony J. Rabbit model for Chlamydia pneumoniae infection. J Clin Microbiol. 1997;35(1):48-52.

8. Muhlestein JB, Anderson JL, Hammond EH, et al. Infection with Chlamydia pneumoniae accelerates the development of atherosclerosis and treatment with azithromycin prevents it in a rabbit model. Circulation. 1998;97(7):633-636.

9. Walsh JH, Peterson WL. The treatment of Helicobacter pylori infection in the management of peptic ulcer disease. $N$ Engl J Med. 1995;333(15):984-991.

10. Saxena VN, Dogra J. Long-term use of penicillin for the treatment of chronic plaque psoriasis. Eur J Dermatol. 2005;15(5):359-362.

11. Taylor-Robinson D, Thomas BJ. Chlamydia pneumoniae in arteries: the facts, their interpretation, and future studies. J Clin Pathol. 1998;51(11):793-797.

12. O'Connor CM, Dunne MW, Pfeffer MA, et al. Azithromycin for the secondary prevention of coronary heart disease events: the WIZARD study: a randomized controlled trial. JAMA. 2003;290(11):1459-1466.

13. Grayston JT, Kronmal RA, Jackson LA, et al. Azithromycin for the secondary prevention of coronary events. $N$ Engl J Med. 2005;352(16):1637-1645.

14. Cannon CP, Braunwald E, McCabe CH, et al. Antibiotic treatment of Chlamydia pneumoniae after acute coronary syndrome. $N$ Engl J Med. 2005;352(16):1646-1654. 
15. Anderson J. Infection, antibiotics, and atherothrombosis - end of the road or new beginnings? N Engl J Med. 2005;352(16):1706-1709.

16. Wolter J, Seeney S, Bell S, Bowler S, Masel T, McCormack J. Effect of long term treatment with azithromycin on disease parameters in cystic fibrosis: a randomized trial. Thorax. 2002;57(3):212-216.

17. Saiman L, Marshall BC, Mayer-Hamblett N, et al. Azithromycin in patients with cystic fibrosis chronically infected with Pseudomonas aeruginosa: a randomized controlled trial. JAMA. 2003;290(13):1749-1756.

18. Oldfield EC 3rd, Fessel WJ, Dunne MW, et al. Once weekly azithromycin therapy for prevention of Mycobacterium avium complex infection in patients with AIDS: a randomized, double-blind, placebo-controlled multicenter trial. Clin Infect Dis. 1998;26(3):611-619.

19. Havlir DV, Dube MP, Sattler FR, et al. Prophylaxis against disseminated Mycobacterium avium complex with weekly azithromycin, daily rifabutin, or both. California Collaborative Treatment Group. N Engl J Med. 1996;335(6):392-398.

20. Saxena VN, Dogra J. Long-term oral azithromycin in chronic plaque psoriasis: a controlled trial. Eur J Dermatol. 2010;20(3):329-333

21. Danesh J, Collins R, Peto R. Chronic infections and coronary heart disease: is there a link? Lancet. 1997;350(9075):430-436.

22. Thom DH, Grayston JT, Siscovick DS, Wang SP, Weiss NS, Daling JR. Association of prior infection with Chlamydia pneumoniae and angiographically demonstrated coronary artery disease. JAMA. 1992;268(1):68-72.

23. Sander D, Winbeck K, Klingelhofer J, Etgen T, Conrad B. Reduced progression of early carotid atherosclerosis after antibiotic treatment and Chlamydia pneumoniae seropositivity. Circulation. 2002;106(19):2428-2433.
24. Vammen S, Lindholt JS, Ostergaard L, Fasting H, Henneberg EW. Randomized double-blind controlled trial of roxithromycin for prevention of abdominal aortic aneurysm expansion. $\mathrm{Br} J \mathrm{Surg}$. 2001;88(8):1066-1072.

25. Wiesli P, Czerwenka W, Meniconi A, et al. Roxithromycin treatment prevents progression of peripheral arterial occlusive disease in Chlamydia pneumoniae seropositive men: a randomized, double-blind, placebo-controlled trial. Circulation. 2002;105(22):2646-2652.

26. Gelfand JM, Neimann AL, Shin DB, Wang X, Margolis DJ, Troxel AB. Risk of myocardial infarction in patients with psoriasis. JAMA. 2006;296(14):1735-1741.

27. Visser MR, Tracy PB, Vercelotti GM, Goodman JL, White JG, Jacob HS. Enhanced thrombin generation and platelet binding on herpes simplex virus-infected endothelium. Proc Natl Acad Sci USA 1988;85(21):8227-8230.

28. Fabricant CG, Hajjar DP, Minick CR, Fabricant J. Herpesvirus infection enhances cholesterol and cholesteryl ester accumulation in cultured arterial smooth muscle cells. Am J Pathol. 1981;105(2):176-184.

29. Lopes-Virella MF, Virella G. Immunological and microbiological factors in the pathogenesis of atherosclerosis. Clin Immunol Immunopathol. 1985;37(3):377-386.

30. Sammalkorpi K, Valtonen V, Kerttula Y, Nikkila E, Taskinen MR. Changes in serum lipoprotein pattern induced by acute infections. Metabolism. 1988;37(9):859-865.

31. Grayston JT. Antibiotic treatment of Chlamydia pneumoniae for secondary prevention of cardiovascular events. Circulation. 1998;97(17):1669-1670.
International Journal of General Medicine

\section{Publish your work in this journal}

The International Journal of General Medicine is an international, peer-reviewed open-access journal that focuses on general and internal medicine, pathogenesis, epidemiology, diagnosis, monitoring and treatment protocols. The journal is characterized by the rapid reporting of reviews, original research and clinical studies across all disease areas.

\section{Dovepress}

A key focus is the elucidation of disease processes and management protocols resulting in improved outcomes for the patient.The manuscript management system is completely online and includes a very quick and fair peer-review system. Visit http://www.dovepress.com/ testimonials.php to read real quotes from published authors. 